\title{
Effects of intravenous ibuprofen and lornoxicam on erythrocyte deformability in rats undergoing hind limb ischemia reperfusion injury
}

\author{
Sivgin $\mathrm{V}^{1}$, Kucuk $\mathrm{A}^{2}$, Comu $\mathrm{FM}^{3}$, Kosem B ${ }^{4}$, Kartal S${ }^{4}$, Turgut $\mathrm{HC}^{5}$, Arpaci $\mathrm{H}^{6}$, Aydin $\mathrm{ME}^{1}$, \\ Koc DS ${ }^{1}$, Ozer $\mathrm{A}^{7}$, Arslan $\mathrm{M}^{1}$, Alkan $\mathrm{M}^{1}$
}

Gazi University Medical Faculty, Department of Anaesthesiology and Reanimation, Ankara, Turkey. mustarslan@gmail.com

\begin{abstract}
BACKGROUND AND AIM: Acute hind limb ischemia reperfusion (I/R) injury is a common consequence of abdominal aorta cross-clamping during aortic surgery. Erythrocyte deformability is affected by I/R process and may lead to increased tissue and organ injury. Lornoxicam and intravenous ibuprofen are becoming commonly used as non-steroidal anti-inflammatory drugs (NSAID) for postoperative analgesia. In this study, we aimed to investigate the effects of lornoxicam (2 mg/kg iv) and intravenous ibuprofen (30 mg/kg iv) on erythrocyte deformability in I/R model in rats. MATERIALS AND METHODS: Four study groups, each containing 6 Wistar rats were created. Laparotomy was performed in all groups under general anesthesia with ketamine and xylazine. In all groups except sham group, ischemia and reperfusion were achieved by clamping and declamping the infrarenal abdominal aorta for 120 minutes. Rats in Group IR+L received intravenous infusion of lornoxicam (2 mg/kg) while rats in Group $\mathrm{IR}+\mathrm{I}$ received intravenous infusion of ibubrofen $(30 \mathrm{mg} / \mathrm{kg})$ following 2 hours of ischemic period. At the end of reperfusion period, erythrocyte packs were prepared from heparinized blood samples. Erythrocyte suspensions with hematocrit at a concentration of $5 \%$ in a phosphate-buffered saline (PBS) were used in order to perform deformability measurements. The value of $p<0.05$ was considered statistically significant.

RESULTS: Relative resistance has increased in ischemia reperfusion group when compared to control group ( $p$ $<0.0001$ ). Lornoxicam or ibuprofen intravenous treatments did not change the erythrocyte deformability during ischemia reperfusion period in rats $(p=0.851, p=0.690)$.

CONCLUSION: Intravenous ibuprofen or lornoxicam administrations during ischemia reperfusion period in rats have no negative effect on erythrocyte deformability. The findings of the study should be supported with more detailed and extensive clinical/experimental studies in the future (Fig. 1, Ref. 18). Text in PDF www.elis.sk. KEY WORDS: ischemia-reperfusion, erythrocyte deformability, intravenous ibuprofen, lornoxicam, rat.
\end{abstract}

\section{Introduction}

Erythrocytes have a biconcave disk of about $8 \mu \mathrm{m}$ in diameter. On the other hand, smallest capillaries of microcirculation are only $3 \mu \mathrm{m}$ in diameter. Beyond these capillaries, the diameter of thick endothelial slits in the spleen is as small as $0.5 \mu \mathrm{m}$ (1).

${ }^{1}$ Gazi University Medical Faculty, Department of Anaesthesiology and Reanimation, Ankara, Turkey, ${ }^{2}$ Dumlupinar University Medical Faculty, Department of Physiology, Kütahya, Turkey, ${ }^{3}$ Kirikkale University Medical Faculty, Department of Physiology, Kirikkale, Turkey, ${ }^{4}$ Turgut Özal University Hospital, Anaesthesiology and Reanimation, Ankara, Turkey, ${ }^{5}$ Gazi University Dentistry Faculty, Department of Oral Maxilla-Facial Surgery, Ankara, Turkey, ${ }^{6}$ Ankara University Dentistry Faculty, Department of Oral Maxilla-Facial Surgery, Ankara, Turkey, and ${ }^{7}$ Gazi University Medical Faculty, Department of Cardiovascular Surgery, Ankara, Turkey Address for correspondence: M. Arslan, Dr, Gazi University Medical Faculty, Department of Anesthesiology and Reanimation, 06510 Ankara, Turkey Phone: +90.312.2026739, Fax: +90.312.2024166

Acknowledgement: The abstract of our manuscript was accepted for oral presentation at the Cardio-Vascular - Thoracic Anaesthesia and Intensive Care Society - 22nd Congress, 2016.
As a consequence, an erythrocyte must deform itself in order to pass through microcirculation of such areas. The extensive and reversible deformation feature of erythrocytes leads to optimal microcirculation of body. The $\mathrm{pH}$ value of the microcirculatory bed, free radicals, surface/volume ratio, proteins of cytoskeleton, viscosity of intracellular environment, hemoglobin concentration, ATP content of cellular stores and cell shape are the main contributors to erythrocyte deformability (2). Sickle cell anemia, hereditary spherocytosis, malaria, septic conditions and vascular diseases may lead to decreased erythrocyte deformability (3). Decreased erythrocyte deformability may result in increased blood viscosity, occlusion in microvessels, impaired perfusion and ischemia. Although underlying mechanisms are partially explained, ischemia-reperfusion affects blood and plasma viscosity, erythrocyte deformability and aggregation $(4,5)$. During and after $\mathrm{I} / \mathrm{R}$ processes, metabolic alterations, inflammatory pathways and excessive free radical generation may result in changes in erythrocyte deformability.

Lornoxicam is a non-steroidal anti-inflammatory drugs (NSAIDs) and belongs to the family of oxicams. Lornoxicam 
has several features including analgesic, antipyretic and antiinflammatory actions. Lornoxicam inhibits the action of COX1 and COX2 enzymes and as a result, it prevents prostaglandin synthesis which plays important roles in inflammatory processes. The anti-inflammatory action of lornoxicam is ten times stronger than that of other oxicam family members such as piroxicam and tenoxicam (6).

Ibuprofen has anti-inflammatory features that limit the inflammatory cascades induced by surgical trauma and reduce the formation of postoperative pain. Intravenous (iv) ibuprofen is a new drug that was first approved in 2009. Primarily, iv ibuprofen was solely used in the management of mild-to-moderate pain. Also the management of moderate-to-severe pain in combination with other analgesics such as narcotics and fever treatment are other frequent indications for this agent (1). Intravenous ibuprofen equally inhibits COX1 and COX2 enzymes. In a different meta-analysis iv ibuprofen provided less morphine requirement and better pain control than placebo does without increased blood loss, renal or respiratory adverse effects and perioperative cardiovascular complications (7). Another advantage of iv ibuprofen is allowing a smooth transition from an intravenous to oral formulation especially for pediatric patients (8).

In this study we aimed to investigate the effects of two NSAID drugs, commonly used in the postoperative period of aortic surgical procedures, on erythrocyte deformability during hind limb ischemia reperfusion injury process in rats.

\section{Materials and methods}

\section{Experimental groups}

After obtaining ethical committee approval from Animal Ethics Committee of Gazi University (GUET 18.02.2016-5828, a total of 24 adult Wistar-albino rats (200-250 gr) were included. The study was conducted in the Physiology Laboratory of Kirikkale University. During the study, the animal care was done in correlation with the standards determined by the National Academy of Science and published by the National Institutes of Health (NIH publication No. 85-23, revised in 1985).

\section{Study design}

Four study groups were created ( $n=6 /$ group). Rats in the sham group (Group S) underwent midline laparotomy and dissection of the infrarenal abdominal aorta (IAA) without ischemia reperfusion process. Rats in ischemia reperfusion group (Group I/R) underwent laparotomy and cross-clamping of the IAA for 120 minutes followed by 120 minutes of reperfusion; in addition to I/R protocol mentioned above, the rats in groups $\mathrm{I} / \mathrm{R}+\mathrm{L}$ and $\mathrm{I} / \mathrm{R}+\mathrm{I}$ received intravenous infusion of lornoxicam ( $2 \mathrm{mg} / \mathrm{kg}$ ) or IV ibuprofen (30 $\mathrm{mg} / \mathrm{kg}$ ), respectively, following 120 minutes of ischemia.

\section{Aortic occlusion and ischemia reperfusion}

General anesthesia was performed using ketamine hydrochloride (Ketalar, $50 \mathrm{mg} / \mathrm{kg}$, intramuscularly, Parke-Davis, Eczacibasi, Istanbul, Turkey) and xylazine hydrochloride (Alfazyne, $2 \%$, Ege Vet, Izmir, Turkey). After fixing the rats in supine position, the abdomen was shaved, and under aseptic conditions midline laparotomy was performed. The abdominal aorta was exposed gently and clamped with an atraumatic microvascular clamp. The aortic occlusion was achieved at the point of losing the distal arterial pulsation. The skin incision was closed and covered with a plastic wrap in order to prevent heat loss and fluid imbalance. After 2 hours of ischemia, the microvascular clamp was removed and lower extremities were reperfused for 2 hours. Following the end of reperfusion period, all rats were sacrificed using ketamine and xylasine. Heparinized blood samples were collected. Erythrocyte suspensions with a hematocrit at $5 \%$ concentration in a phosphate-buffered saline (PBS) were used in order to perform deformability measurements.

\section{Deformability measurements}

Blood samples from rats were taken and sufficient measurements were performed as fast as possible in order to prevent hemolysis of erythrocytes. Blood samples were centrifuged at 1000 rpm for ten minutes. Serum and buffy coat on erythrocytes were removed. After addition of isotonic PBS buffer on to the collapsing erythrocytes, centrifugation was performed at a rate of 1000 rpm for ten minutes. Liquid on the upper surface was removed. Subsequently pure red cell packs obtained from the washing process were mixed with the PBS buffer in order to achieve a suspension with a concentration level of $5 \%$ hematocrit. Deformability measurements were performed using those suspensions. All the aforementioned processes were performed at $22^{\circ} \mathrm{C}$.

Ten milliliters of erythrocyte suspension plus PBS buffer were used in order to perform deformability measurements via a filtrometer system. A flow rate of $1.5 \mathrm{ml} / \mathrm{min}$ was achieved using an infusion machine. Pressure changes were recorded via a pressure transducer. The obtained data were processed and calculations were made using a special software program (Biopac Systems Inc, Commat, USA). The relative refractory period value (Rrel) is an important parameter in deformability measurements and was calculated using the pressure values of suspension to the pressure value of buffer. The deformability index determined as Rrel was increasing the ability of erythrocytes to deform themselves. Accelerating Rrel values were inversely correlated with the erythrocytes' deformability (9).

\section{Statistical analyses}

Statistical Package for the Social Sciences (SPSS, Chicago, IL, USA) 17.0 program was used for statistical analysis. KolmogorovSmirnov test was used to compare the distributions of variable groups. Kruskal-Wallis test was performed in order to measure deformability variations. Bonferroni adjusted Mann-Whitney U test was used for assessing intergroup differences after Kruskal-Wallis test. Results were expressed as mean \pm standard deviation (mean \pm $\mathrm{SD})$. The value of $\mathrm{p}<0.05$ was considered statistically significant.

\section{Results}

Relative resistance which indicates decreased erythrocyte deformability has increased in the ischemia reperfusion group $(p$ 


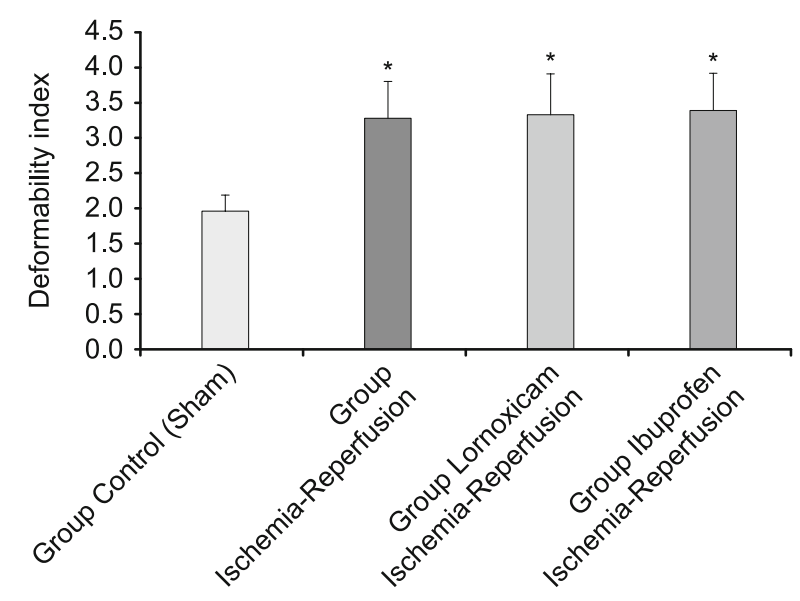

Fig. 1. Data from erythrocytes deformability of the study groups. Each bar represents the mean $\pm \mathrm{SD} .{ }^{*} \mathbf{p}<0.05$ compared to the Group Control.

$<0.0001)$. Neither lornoxicam nor ibuprofen treatment changed the erythrocyte deformability in rats that underwent $\mathrm{I} / \mathrm{R}$ injury ( $\mathrm{p}$ $=0.851 ; \mathrm{p}=0.690)$ (Fig. 1).

\section{Discussion}

Aortic cross-clamping is commonly performed during cardiovascular surgery. The ischemic tissue injury after cross-clamping and subsequent declamping is a common consequence of aortic surgery. Also reperfusion of ischemic areas facilitates local and systemic complications which may result in increased mortality and morbidity. Systemic inflammatory response syndrome (SIRS) and multiple organ failure (MOF) are two important consequences of $\mathrm{I} / \mathrm{R}$ injury $(10,11)$. In these circumstances, amelioration of blood flow is essential in order to avoid cell death and deterioration of the ischemic damage by I/R injury (12).

The rheological parameters of erythrocytes, such as deformability and aggregation are substantially affected by ischemiareperfusion process. Reactions triggered by inflammation, hemodynamic changes, generation of free-radicals and mediators, acute phase reactions and alterations in the coagulation cascade can further aggravate microcirculatory deterioration. Decreased erythrocyte deformability leads to microcirculatory disarrangements such as capillary occlusion and diminished oxygen delivery for the whole body (13). In this manner, the examination of erythrocyte deformability may ensure important information on circulation and free radicals during I/R injury (14).

Oxicams are a new family of NSAIDs belonging to the group of enolic acids. Piroxicam, meloxicam, and tenoxicam are similar in structure, albeit with a different ring. In order to reduce side effects related to gastrointestinal system, lornoxicam, cinnoxicam, and sudoxicam have been developed. Oxicams are mainly used in the treatment of arthritis and postoperative inflammation (6).

There are limited data regarding the effects of lornoxicam during I/R injury. In one study Gavrilova et al (15) showed that single intravenous injection of $230 \mathrm{mg} / \mathrm{kg}$ of lornaxicam $20 \mathrm{~min}$ after the onset of ischemia reduced the injury demonstrated by decreased volume of necrosis and degree of the thinning of the left ventricular wall in the region of infarction, and lowered the volume of connective tissue in the myocardium. To our knowledge our previous study is the only study investigating the effects of lornoxicam on erythrocyte deformability (16). In this study, lornoxicam failed to improve the tissue perfusion with decreased blood flow and erythrocyte deformability (16). In contrast to the latter study, at least we found erythrocyte deformability unchanged with lornoxicam during I/R injury. We suggest that results opposing those from other studies indicate the need for further evaluations while focusing on the connection between the local and systemic rheological changes during ischemia-reperfusion.

Our pubmed search using ibuprofen iv, I/R and erythrocyte deformability as keywords resulted in finding only two studies. In the study conducted by Cole et al (17), the authors investigated the effects of ibuprofen iv on focal cerebral ischemia. In this study, Cole et al (17) found a reduced brain infarction volume in animals treated with iv ibuprofen when reperfusion was allowed. On the other hand, in animals with permanent middle cerebral artery (MCA) occlusion, ibuprofen worsened the infarction volume. The authors suggested that the beneficial effect of cyclooxygenase inhibition occurs during reperfusion. We think that this finding is important because both ibuprofen and lornoxicam treatments during reperfusion period in our study did not result in worsened erythrocyte deformability. In addition, Cole et al were not able to explain the result of their study. Nevertheless, they acknowledged the possibility that ibuprofen might have crossed the blood-brain barrier more readily after MCA occlusion than in normal situations from which the disposition kinetic profile was obtained. Also they suggested that only intravascular inhibition of cyclooxygenase at the endothelial cell, and not the intraparenchymal inhibition, could be necessary to achieve the reduction in infarction volume. 17

In other study, Bozzo et al (18) treated the blood samples with different NSAIDs (aspirin, dipyrone, ketorolac, ibuprofen) and found that erythrocyte deformability was statistically similar to that of control in all treatment groups (dipyrone, $0.80 \pm 0.07$; ibuprofen, $0.83 \pm 0.04$; ketorolac, $0.85 \pm 0.05$; aspirin, $0.56 \pm 0.05$ ). However they found that aspirin treatment significantly reduced erythrocyte deformability compared to dipyrone, ibuprofen and ketorolac $(\mathrm{p}<0.05)$. Also they reported that in all study groups which received NSAIDs, the typical discoid shape of erythrocytes was unaffected (18). Although not conducted as an I/R model, the results of this study strongly support our findings.

In conclusion, we found that both of NSAID drugs -intravenous ibuprofen and lornoxicam- did not worsen erythrocyte deformability during aortic I/R period. This result suggests that an administration of two drugs for postoperative pain can be safe and harmless - at least in terms of erythrocyte deformability. The findings of this study need to be supported in the future by extensively designed animal and human studies. 


\section{References}

1. Viallat A, Abkarian M. Red blood cell: from its mechanics to its motion in shear flow. Int J Lab Hematol 2014; 36 (3): 237-243.

2. Fisher T, Stohr-Liesen M, Schmid-Schonbein H. The red cell as a fluid drop let: tank tread-like motion of the human erythrocyte membrane in shear flow. Science 1978; 202: 894-896.

3. Yedgar S, Koshkaryev A, Barshtein G. The red blood cell in vascular occlusion. Pathophysiol Haemost Thromb 2002; 32: 263-268.

4. Khairy K, Howard J. Minimum-energy vesicle and cell shapes calculated using spherical harmonics parameterization. Soft Matter 2011; 7: 2138-2143.

5. Fisher TM. Shape memory of human red blood cells. Biophys J 2004; 86: 3304-3313.

6. Tsakiridis K, Mpakas A, Kesisis G, Arikas S, Argyriou M, Siminelakis $\mathbf{S}$ et al. Lung inflammatory response syndrome after cardiac-operations and treatment of lornoxicam. J Thorac Dis 2014; 6 (S1): S78-S98.

7. Southworth SR, Woodward EJ, Peng A, Rock AD. An integrated safety analysis of intravenous ibuprofen (Caldolor $($ ) in adults. J Pain Res 2015; 8: 753-765.

8. Moss R, Watcha MF, Bendel LP, McCarthy DL, Witham SL, Glover CD. A multicenter, randomized, double-blind placebo-controlled, single dose trial of the safety and efficacy ofintravenous ibuprofen for treatment of pain in pediatric patients undergoing tonsillectomy. Pediat Anesth 2014; 24: 483-489.

9. Arslan M, Comu FM, Işik B, Unal Y, Cekmen N, Kurtipek O. Effects of a general anaesthetic agent, propofol, on erythrocyte deformability. Bratisl Med J 2010; 111: 126-128.

10. Duru S, Koca U, Oztekin S, Olguner C, Kar A, Coker C et al. Antithrombin III pretreatment reduces neutrophil recruitment into the lung and skeletal muscle tissues in the rat model of biletaral lower limb and reperfusion: A pilot study. Acta Anaesthesiol Scand 2005; 49: 1142-1148.
11. Turchányi B, Tóth B, Rácz I, Vendégh Z, Furész J, Hamar J. Ischemia reperfusion injury of skel-etal muscle after selective deafferentiation. Physiol Res 2005; 54: 25-32.

12. Rocha Bda C, Mendes RR, Lima GV, Albuquerque Gde S, Araújo LL, de Jesus MN et al. Experimental model of mesenteric ischemia/reperfusion by abdominal aorta clamping in Wistar rats. Rev Col Bras Cir 2012; 39 (3): 207-210.

13. Baskurt OK, Meiselman HJ. Blood rheology and hemodynamics. Semin Thromb Hemost 2003; 29 (5): 435-450.

14. Nemeth N1, Lesznyak T, Szokoly M, Furka I, Miko I Allopurinol prevents erythrocyte deformability impairing but not the hematological alterations after limb ischemia-reperfusion in rats. J Invest Surg 2006; 19 (1): 47-56.

15. Gavrilova SA, Lipina TV, Zagidullin TR, Fominykh ES, Golubeva AV, Varenik EN et al. Protective effect of lornoxicam on development of myocardial infarction in rats under conditions of ischemia and ischemiareperfusion. Kardiologiia 2008; 48 (12): 42-48.

16. Arslan M, Comu FM, Isik B, Atac MS, Yilmaz D. The evaluation of effects of lornoxicam on blood flow and erythrocyte deformability in comparison with iv paracetamol in rats. Bratisl Lek Listy 2012; 113 (4): 211-213.

17. Cole DJ, Patel PM, Reynolds L, Drummond JC, Marcantonio S. Temporary focal cerebral ischemia in spontaneously hypertensive rats: the effect of ibuprofen on infarct volume. J Pharmacol Exp Ther 1993; 266 (3): 1713-1717.

18. Bozzo J, Escolar G, Hernández MR, Galán AM, Ordinas A. Prohemorrhagic potential of dipyrone, ibuprofen, ketorolac, and aspirin: mechanisms associated with blood flow and erythrocyte deformability. J Cardiovasc Pharmacol 2001; 38 (2): 183-190.

Received August 7, 2016. Accepted September 19, 2016. 